\title{
Hybrid Intelligent Algorithm Solving Uncertainty Job-Shop Scheduling Problem
}

\author{
Yang-Jun $\mathrm{HU}^{1}$, Cun-li SONG ${ }^{2}$ \\ School of Dalian Jiao Tong University, Dalian 116052, China. \\ ${ }^{1} 1057240154 @ q q . c o m,{ }^{2}$ scunli@163.com
}

Keywords: Job shop scheduling; fuzzy mathematics; immune; taboo

\begin{abstract}
Researched the uncertain Job-Shop Scheduling, on the basis of the original triangular fuzzy number to describe fuzzy processing time, structured the fuzzy Job-Shop Scheduling model. Algorithm using the concept of "big valley" topology represent solution space, using strong swap mutations in early immune genetic algorithm, and implanting vaccines in three styles, rapidly improved the ability of search "mountain"; After immune selection using taboo search’s "climb" idea improve the local search ability of the algorithm, so as to choose the individual with maximum satisfaction in the "big valley" quickly and efficiently. And through Matlab2012a software simulation examples verify the effectiveness of the immune genetic and taboo hybrid intelligent algorithm.
\end{abstract}

\section{Introduction}

Job-Shop Scheduling Problem has always been one focus of Researchers who research scheduling direction. Uncertain Job-Shop Scheduling Problem, due to its wide range of uncertainties and more in line with actual production environment, having great theoretical and practical significance.

People usually solve uncertainties into three kinds of academic approach: First, Random Method, involving disciplines Probability Theory and Mathematical Statistics, mainly used to deal with the objects without defined mappings, strong random features and unreliability of human behavior. Second, Fuzzy Method, based on fuzzy sets[1], mainly dealing with the Vagueness and uncertainty of data and information. GENG[2] using the theory of Six fuzzy numbers to establish Uncertain Job-Shop Mathematical Model, Genetic Algorithm to solve the optimal scheduling order; YUAN[3], respectively using triangular and trapezoidal fuzzy numbers to indicate uncertainty Processing Time and delivery. Third, other methods, such as Rough Set Theory, Grey System Theory, Confidence Network, etc. mainly involved in other types of uncertain information research.

Because of Fuzzy Set Theory closer to the actual production scheduling situation, we use Fuzzy Theory describe the resource constraints, process constraints and environmental constraints of Uncertain Job-Shop Scheduling. Its idea use triangular fuzzy numbers to describe the whole fuzzy time in process, the average customer satisfaction to measure the fitness of the antibody, the Improved Immune Genetic and Taboo Search Hybrid Algorithm to solve the Uncertain Job-Shop Scheduling Problem, then by the example to verify the correctness and effectively of the algorithm.

\section{The Description of Uncertainty Job-Shop Scheduling Problem}

For now, Job-Shop Scheduling Problems generally limited to ideal determined conditions, but uncertainty interference from personnel, equipment and environment is especially, they make related solutions including Components of the problem can not be clearly identified. With in-depth study of Fuzzy Set Theory, people found it is possible to combine uncertain time parameter with Fuzzy Sets Theory, and membership function characterize Job-Shop scheduling problem uncertainties. This article is based on this view, uses fuzzy numbers describe these time parameters and delivery of Uncertain Job-Shop Scheduling, have a profound theoretical significance and wide application value. 


\section{Mathematical Model of Uncertain Job-Shop Scheduling}

Uncertain Job-Shop Scheduling Problem can be described as follows: N jobs will be processed on $\mathrm{M}$ devices in a certain order, all processes Priorities of all works, all processes fuzzy operation time and delivery time are given in advance, the order we arrange is each workpiece on each operation of the device and without disrupting the work given step Priorities and time constraints, make the average customer satisfaction to achieve optimal. The ${ }^{c_{i, j, k}}$ represents the completion time of the $\mathrm{j}$-procedure of $\mathrm{i}$-workpiece on the machine $\mathrm{K}$, the ${ }^{p_{i, j, k}}$ represents the operating time of the $\mathrm{j}$-procedure of i-workpiece on the machine K, and constraints of the scheduling model are as follows:

(1) Each processing station at a particular time can only process one workpiece;

(2) Once all steps processing, no possibility of interruption;

(3) The j-procedure of i-workpiece must be start at the completion of its (j-1)-procedure, namely $c_{i, j, k}-p_{i, j, k} \geq c_{i, j-1, k}(j \geq 2$,int $)$

(4) each workpiece at each time can only processing on one device, such as $p_{i, j, k}$, when the $\mathrm{i}$ and $\mathrm{j}$ is determined,the $\mathrm{k}$ must is a value in set $\{1,2, \ldots, \mathrm{m}\}$ or an empty set.

It is worth noting that ${ }^{p_{i, j, k}}$ and ${ }^{c_{i, j, k}}$ are triangular fuzzy number.Operation between the two fuzzy numbers involved whichever is greater, whichever is less and other operations, calculation is more complicated.

\section{Fuzzy Numbers and Processing Time}

The concept of triangular fuzzy, membership function describes as follows:

$$
u(x)=F_{\tilde{A}}(x)=\left\{\begin{array}{cc}
\frac{x-a_{1}}{a_{M}-a_{1}} & \left(a_{1} \leq x \leq a_{M}\right) \\
\frac{x-a_{2}}{a_{M}-a_{2}} & \left(a_{M} \leq x \leq a_{2}\right) \\
0 & \text { (otherwise) }
\end{array}\right.
$$

As above, it called $\tilde{A}$ as triangular fuzzy number, $\left[a_{1}, a_{2}\right]$ is a variable interval, the point ${ }^{\left(a_{M}, 1\right)}$ is the most value. Fuzzy numbers shown as Figure 1.1 is a geometric representation of fuzzy numbers above, it can be written as $\tilde{A}=\left(a_{1}, a_{M}, a_{2}\right)$.

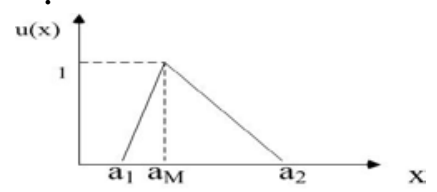

Fig 1.1 Triangular Fuzzy Numbers Represent the Uncertain Processing Time

This article use triangular fuzzy number describe the expected completion time and delivery. When represent fuzzy time, $a_{1}, a_{M}$ and ${ }^{a_{2}}$ represent the most optimistic, the maximum possible and the most pessimistic value respectively, three values here is a percentage of the use of time of the actual production process, if the time $\mathrm{T}$ appears more often, then $\mathrm{T}$ is the maximum possible value of fuzzy time period. When represents delivery, can be written as $\tilde{D}=\left(a_{1}, a_{M}, a_{2}\right)$, and $a_{M}=\frac{a_{1}+a_{2}}{2}$.

In the study of this title, fuzzy numbers operation is particularly important, all previous steps must take a large operation for the fuzzy time before the operation; when all steps completed, need summing operation for the fuzzy numbers; decoding need compare operation for fuzzy. The test is mainly related to the addition, comparison, and whichever is greater Operation of fuzzy number. The first two can be seen $\tilde{A}=\left(a_{1}, a_{2}, a_{3}\right)$ as a vector, that is vector addition and whichever is greater Operation, and the compare operation described here in relates to the use of three criteria of Masatoshi Sakawa [4], here not to repeat. 


\section{Immune Taboo Search Algorithm for Uncertain Job-Shop Scheduling Problem}

Uncertain Job-Shop Scheduling requires not only fuzzy conditional modeling, also need to carry out a variety of complex operations for fuzzy numbers, more complex and challenging than the traditional Job-Shop Scheduling.

\section{Improved Immune Taboo Search Algorithm for Uncertain Job-Shop Scheduling}

Because of Fuzzy Set Theory closer to the actual production scheduling situation, we use Fuzzy Theory describe the resource constraints, process constraints and environmental constraints of Uncertain Job-Shop Scheduling.

For the characteristics of the problem, author construct a fuzzy mathematical model, taking into account the evolution features of the immune genetic algorithm is slow in the early time and precocious easily in the late stage,by the use of three forms inoculation of vaccination, and in the early evolution use strong exchange variation mutations, the plug-in variation with less change of transformation step sequence in late period. Increase the rate of population early evolution. The average customer satisfaction to measure the fitness of the antibody, the Improved Immune Genetic and Taboo Search Hybrid Algorithm to solve the Uncertain Job-Shop Scheduling Problem, then by the example to verify the correctness and effectively of the algorithm.Algorithmic process shown in Figure 2.1:

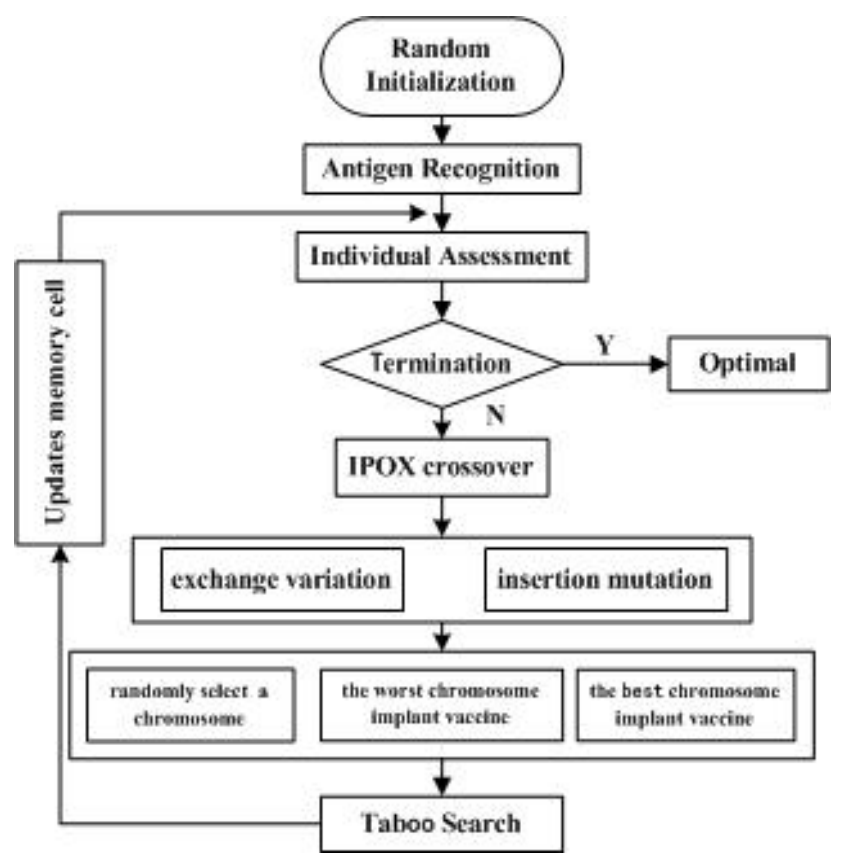

Fig 2.1 Improved Immune Taboo Algorithm Flowchar

\section{Antigen Recognition and Individual Assessment}

Antigen recognition is the optimization objectives and some constraints input as an antigen. In this paper, the objective function is the average maximum satisfaction of customers.

$\mathrm{AI}$, satisfaction, is one of most directly concerned evaluation of customers and processing plant in fuzzy scheduling problem. It has the direct impact of costs and customer credibility, and its visual representation is the degree of overlap in the range of job completion and customer desired delivery, has important economic significance and practical value. Therefore, using the average customer satisfaction measure the satisfaction of all of the workpiece in scheduling process. Have

$$
f_{1}=\max \left(\frac{1}{n} \sum_{j=1}^{n} A I_{j}\right)
$$

${ }^{A I_{j}}{ }$ is the satisfaction of the $\mathrm{j}$-workpiece relative to the delivery,

$$
A I_{j}=\frac{\tilde{C}_{j} \cap \tilde{D}_{j}}{\tilde{C}_{j}}
$$


$\tilde{C}_{j}$ represents the completion fuzzy time of the job $\mathrm{j}$ and ${ }^{\tilde{D}_{j}}$ represents the client's expectations delivery of the job $\mathrm{j}$. That satisfaction is the ratio of completion time within the delivery with the entire fuzzy completion time, also is the the ratio of shadow in Figure 2.2 with the whole:

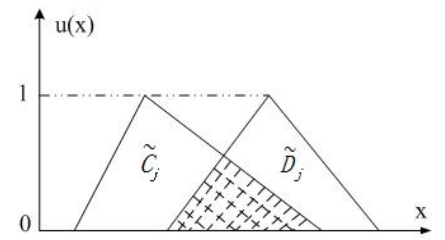

Fig 2.2 The Depiction of Satisfaction Graphics

Fitness function is the basis for the choice in Algorithm optimization process, its definition has a direct impact on the efficiency of the optimization algorithm. Fitness function is the objective function in here.

\section{Encoding and Decoding}

In this paper, encoding use the more common chromosomal coding method which is based on step, Greed Thought to realize the decoding process. The process is as follows: First step in accordance with the order on the resulting sequence to decoded and then each process in the equipment process as high as possible, decoding until all work arrangements in best position.

\section{Initialization}

While Taboo Search depends heavily on the merits of the initial solution, but Immune Genetic Algorithm who play a major role has increased the excellent quality, and the initialization improvements may increase the complexity of the algorithm complexity is not conducive to scheduling solution search, and therefore randomly generated the first generation population.

\section{Immune Genetic Manipulation}

Crossover. Algorithm using IPOX crossover. The basic procedure is as follows: Firstly, the job randomly divided into two sets G1 and G2, copy the job of parent antibody-1 which contained in the G1 to child-1, copy the job of parent antibody-2 which contained in the G2 to child-2, and retain the original location of these job; then, copy the job of parent antibody- 1 which contained in the G1 to child-2, copy the job of parent antibody-2 which contained in the G2 to child- 1 , and retain the original location of these job. Thereby, obtain new antibodies.

Mutation Operator. Glover[5] have used the GATS hybrid algorithm to test several commonly variation, such as insertion mutation, exchange variation and reverse mutation, showed that exchange variation have more excellent performance, therefore this article use exchange variation before the early iteration,that is arbitrarily select two loci ${ }^{v_{1}, v_{2}}$, place ${ }^{v_{1}}$ in position on ${ }^{v_{2}}$, place ${ }^{v_{2}}$ in position on

$v_{1}$, change their respective positions, to achieve the purpose of strong mutation, increase convergence rates in the early time. When the late of iterations, use the insertion mutation, namely, randomly select a gene and randomly insert a gene locus in the chromosome, appropriate to reduce the rate of convergence, and enhance the ability of local search. Thereby generating a new individual.

Immune Operator. In order to improve the convergence rate, this title uses the principle of the Shortest Processing Time to extract vaccine. Three inoculation methods of the vaccine has. First, randomly select a chromosome implant vaccine. Second, the worst chromosome implant vaccine. Third, the best chromosome implant vaccine. As we all know, many types of vaccinations manner, it is possible to increase the probability of chromosomes reach the optimal solution, so as to avoid the loss of the optimal solution.

\section{Vaccination and Immune Memory}

Antibody implant vaccine in the corresponding alleles. First of all, extract vaccine on the base of the principle of the Shortest Processing Time, constitute the gene segment; Then, delete the contents of loci on the corresponding allele, and implant the corresponding vaccine into it, generate new antibody.

Immunological memory involved in updating the population in the form of memory cells, comes into play in the second generation. That is the second generation of the population is part of randomly generated population and some antibodies which in memory unit. Update memory unit is equally 
critical population evolution, when a high fitness individuals appear and never appear in the memory unit, then to replace the current worst individual in the memory unit, thereby enhancing the efficiency of the algorithm.

\section{Taboo Search}

In the memory cell select an optimal solution into taboo search, by changing the neighborhood structure, choose the antibody who is superior than its parent to replace the worst individual in the memory cells, rather than replace the parent, and move the moving property into taboo table. Worthy of note here is that although the taboo table is a memory unit, but is different from the immune memory cells, the former is a mobile property of chromosome, and the later is chromosome itself, whether physical or logical location positions are separate. Proceed as follows:

1) Select an optimal solution in antibodies denoted $s^{*}$, fitness $f\left(s^{*}\right)$;

2) Structural changes in the neighborhood generate neighborhood Solutions, if $s^{*}$ is the best, memory unit unchanged, taboo algorithm terminates;

3) If it is better than $s^{*}$, or meets the amnesty criteria and not to taboos, then replaces the current solution; and presents the optimal solution itself to the memory unit, replaces the worst solution; and replaces mobile property into taboo table;

4) If the new solution ${ }^{s}$, and $f(s) \leq f\left(s^{*}\right)$,then returns to the step (2).

\section{Antibody population updates}

The update is divided into two steps of memory unit, Step 1, After selection, there are some antibodies reserved into the memory unit; step 2, With Taboo search algorithm to judge whether the current best solutions is still has the better one in the current memory unit, if has, update it, if not just the same.

\section{Termination and The Optimal Solution}

Determine whether the termination condition is satisfied, that is, whether the maximum number of iterations or optimal solution appears. If satisfied, the algorithm terminates, outputs the optimal scheduling solution; otherwise, return to step 4.

\section{Simulation Example}

\section{Simulation Environment}

In AMD, CPU 999MHz, 1.74GB RAM, Windows XP operating system, Using Matlab2012a simulation tools to achieve the above algorithm. Here the date is from Yiwu ZQ handicraft processing plant and the initial parameters of the algorithm is: population size is 20 , the maximum number of iterations is 100 , IPOX crossover probability is 0.8 , pre-mutation probability and late-mutation probability is 0.02 , Vaccination probability is 0.08 , memories storage capacity is 10 , taboo table is 5 .

Process, fuzzy processing time and machine constraints such as Table 1, can be described as 8 job on 10 machines, each processing time of the step are fuzzy. Specifically described as follows: The first step of $\mathrm{J} 1$ takes $(1,3,4)$ machining time on the machine 3, the delivery of J1 is[31,55] .

\section{Simulation Results}

The results obtained by simulation results were compared with the actual delivery, respectively, as shown in Tables 2 and 3:

Table 1 Step - Fuzzy processing time - Machine constraints

\begin{tabular}{cccccccc}
\hline Work & Step 1 & Step 2 & Step 3 & Step 4 & Step 5 & Step 6 & Deadline \\
\hline $\mathrm{J}_{1}$ & $(1,3,4) 3$ & $(8,10,12) 1$ & $(7,9,11) 2$ & $(3,5,6) 4$ & $(2,3,4) 8$ & $(8,10,12) 3$ & {$[31,55]$} \\
$\mathrm{J}_{2}$ & $(4,6,8) 2$ & $(7,8,9) 3$ & $(1,1,2) 5$ & $(4,5,6) 6$ & $(2,3,5) 1$ & $(1,3,4) 10$ & {$[28,45]$} \\
$\mathrm{J}_{3}$ & $(1,1,2) 3$ & $(4,5,7) 4$ & $(3,5,6) 6$ & $(4,5,6) 1$ & $(7,9,11) 2$ & $(1,1,2) 5$ & {$[25,45]$} \\
$\mathrm{J}_{4}$ & $(5,7,9) 4$ & $(1,3,5) 9$ & $(3,4,5) 3$ & $(1,3,4) 4$ & $(1,1,2) 5$ & $(2,3,5) 6$ & {$[29,47]$} \\
$\mathrm{J}_{5}$ & $(5,6,7) 5$ & $(8,10,12) 2$ & $(6,7,8) 3$ & $(7,8,9) 9$ & $(3,5,6) 1$ & $(2,4,6) 4$ & {$[37,53]$} \\
$\mathrm{J}_{6}$ & $(2,3,4) 2$ & $(9,10,12) 4$ & $(5,7,9) 9$ & $(8,9,10) 1$ & $(3,4,6) 5$, & $(8,9,10) 3$ & {$[38,54]$} \\
$\mathrm{J}_{7}$ & $(3,4,6) 7$ & $(5,7,9) 10$ & -- & -- & -- & -- & {$[10,30]$} \\
$\mathrm{J}_{8}$ & $(2,3,4) 9$ & $(1,3,5) 7$ & $(3,4,5) 8$ & -- & -- & -- & {$[6,20]$} \\
\hline
\end{tabular}


Table 2 Internal delivery of Production task

\begin{tabular}{cccc}
\hline Work & Delivery & Work & Delivery \\
\hline $\mathrm{J}_{1}$ & on time & $\mathrm{J}_{5}$ & on time \\
$\mathrm{J}_{2}$ & 1 hour ahead & $\mathrm{J}_{6}$ & delayed 10 hours \\
$\mathrm{J}_{3}$ & on time & $\mathrm{J}_{7}$ & on time \\
$\mathrm{J}_{4}$ & 3 hour ahead & $\mathrm{J}_{8}$ & on time \\
\hline
\end{tabular}

Table 3 The Comparison of Two Kinds of Immune Genetic Algorithm based on Fuzzy Scheduling

\begin{tabular}{|c|c|c|c|c|c|c|c|c|}
\hline W & \multicolumn{4}{|c|}{ Immune Genetic Algorithm(IGA) } & \multicolumn{4}{|c|}{ Improved Immune Genetic Algorithm(IIGA) } \\
\hline $\begin{array}{l}\text { or } \\
k\end{array}$ & $\begin{array}{c}\text { Completion } \\
\text { Time(CT) }\end{array}$ & Min-CT & $\begin{array}{c}\text { Satisfact } \\
\text { ion(S) }\end{array}$ & Avg-S & $\begin{array}{c}\text { Completio } \\
\text { n Time }\end{array}$ & Min-CT & $\begin{array}{c}\text { Satisfa } \\
\text { ction }\end{array}$ & Avg-S \\
\hline $\mathrm{J}_{1}$ & $(34,46,57)$ & & 0.9842 & & $(43,52,63)$ & & 0.7091 & \\
\hline $\mathrm{J}_{2}$ & $(25,31,38)$ & & 0.8846 & & $(27,35,44)$ & & 0.9926 & \\
\hline $\mathrm{J}_{3}$ & $(29,38,55)$ & & 0.7738 & & $(38,47,59)$ & & 0.2593 & \\
\hline $\mathrm{J}_{4}$ & $(22,29,39)$ & $(41,55,67$ & 0.5882 & 08163 & $(30,34,52)$ & $(43,52,63$ & 0.9369 & 08369 \\
\hline $\mathrm{J}_{5}$ & $(32,43,59)$ & ) & 0.8325 & 0.8163 & $(35,46,58)$ & ) & 0.8778 & 0.8369 \\
\hline $\mathrm{J}_{6}$ & $(41,55,67)$ & & 0.4643 & & $(35,42,51)$ & & 0.9196 & \\
\hline $\mathrm{J}_{7}$ & $(11,17,24)$ & & 1.0000 & & $(11,17,24)$ & & 1.0000 & \\
\hline $\mathrm{J}_{8}$ & $(6,10,16)$ & & 1.0000 & & $(6,10,16)$ & & 1.0000 & \\
\hline
\end{tabular}

Table 2 shows, Most of the work inside the plant can be completed on time, but poor punctuality, customer satisfaction reached only 0.625 .

Improved algorithm on the base of Immune Genetic Algorithm, average maximum satisfaction for the optimization goal, and both results were compared in Table 3. It is obviously concluded that both results are much better than the actual data in Table 2 . The improved algorithm significantly better than the former in completion time, and there was a marked increase in satisfaction, the validity of the algorithm in uncertain optimization. Figure 3.1 corresponds to the evolution of the curve. Due to the improved algorithm varied greatly in early, the optimal solution much better than the optimal solution of traditional Immune Genetic Algorithm. Although the optimal solution convergence rate from 24 to 30 generations is weaker than traditional Immune Genetic Algorithm, jump out of local optimum quickly, to achieve global optimization purposes. The Gantt of optimal solution is shown in Figure 3.2 , one of the most optimized sequence is

1-5-6-8-7-1-2-3-6-8-7-8-5-2-4-6-2 -2-1-5-6-3-4-4-3-5-2-1-6-3-2-6-1-4-4-5-3-4-1-5-3.

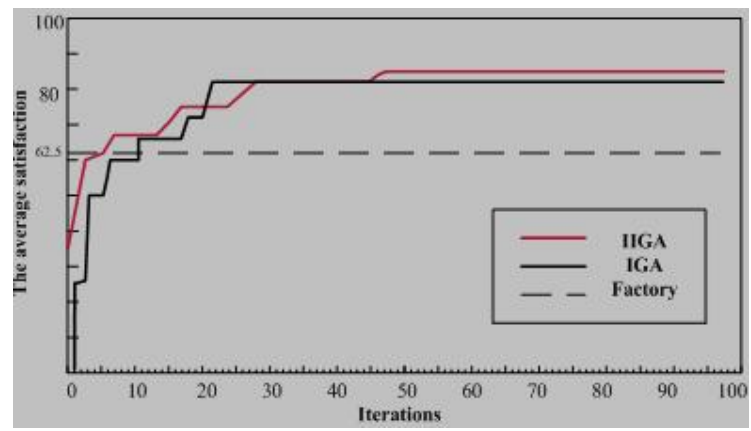

Fig 3.1 Improved Immune-Taboo Convergence Graph

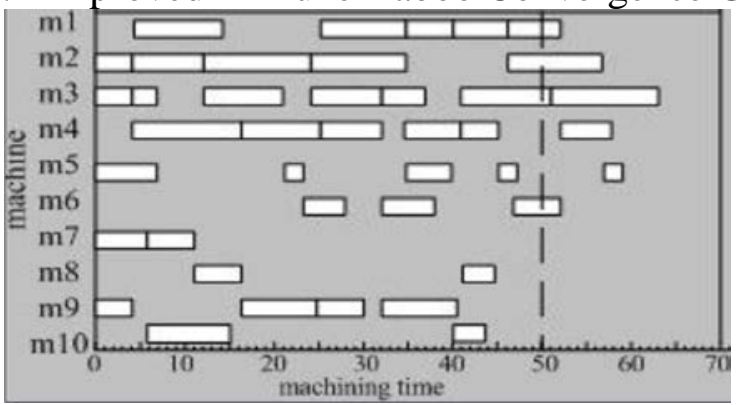

Fig $3.28 \times 10$ Job-Gantt Graph 


\section{Conclusion}

The Job-Shop Scheduling under uncertainty compared to conventional deterministic Job-Shop S cheduling more realistic on production scheduling, has practical guiding significance.This title made a mathematical simulation for fuzzy time firstly, established a Job-Shop Scheduling mathematical model under conditions of uncertainty, designed a improved Immune Taboo search algorithm which has exchange mutation operator and insertion mutation operator , three ways of implanting vaccine and the basic process is given to verify the effectiveness of the immune taboo algorithm.

However, the fuzzy processing time which represented by the triangular fuzzy number and the actual time is still have a certain gap, and the uncertainty of time caused by the urgent arrival, machine failure in real life, will be explored in future research.

\section{References:}

[1] ZADEH L A. Fuzzy sets[J]. Information and Control, 1965, 8(3): 338-353.

[2] Zhao-Qiang GENG, Yi-Ren ZHOU. Fuzzy Job Shop Scheduling Problem Based on Genetic Algorithms[J]. Computer Integrated Manufacturing Systems, 2002, 8 (8).

[3] Bo YUAN, Bao-Sheng YING, Hao XIE. Based on Genetic Algorithm under Uncertain Conditions for Job Shop Scheduling [J]. Modern Manufacturing Engineering, 2012, 10: 52-56.

[4] MASATOSHI S,RYO K. Fuzzy Programming for Multi-Objective Job Shop Scheduling with Fuzzy Processing Time and Fuzzy Due Date through Genetic Algorithm[J]. European Journal of Operational Research, 2000, 120(2): 393-407.

[5] GLOVER F, KELly J, LAGUNA M. Genetic Algorithm and Tabu Search: Hybrids for Optimization[J]. Computers and Operations Research, 1995, 22(1), 111-134.

Yang-Jun HU(1990-),female, graduate students, mainly research is intelligent algorithms and shop scheduling; E-mail: 1057240154@qq.com;

Cun-Li SONG(1975-), female, Ph.D., associate professor, mainly research is shop scheduling and intelligent optimization algorithms; E-mail: scunli@163.com. 